\title{
Gender Differences in Functional Connectivity during Emotion Regulation
}

\author{
Authors: Stoica, T. ${ }^{* 1}$, Knight, L.K. ${ }^{1}$, Naaz, F. ${ }^{2}$, Patton, S.C. ${ }^{1,3}$, Depue, B.E. ${ }^{1,4}$ \\ ${ }^{1}$ University of Louisville, Department of Psychological and Brain Sciences, $2301 \mathrm{~S} 3^{\text {rd }} \mathrm{St}$ \\ Louisville, KY 40292 \\ 2Johns Hopkins University School of Medicine, Department of Psychiatry and Behavioral \\ Sciences, 1800 Orleans St, Baltimore MD 21287 \\ ${ }^{3}$ Southeast Louisiana Veterans Health Care System, 2400 Canal St, New Orleans, LA \\ 70119 \\ ${ }^{4}$ University of Louisville, Department of Anatomical Sciences and Neurobiology \\ *Corresponding Author: teodora.stoica@louisville.edu
}

\section{Abstract}

Gender differences in emotion regulation (ER) have been postulated, yet their neural basis remains poorly understood. The goal of this study was to investigate this issue from a functional connectivity (FC) perspective. Utilizing a region of interest (ROI) analysis, we investigated whether men and women $(\mathrm{N}=48)$ differed in their FC pattern while viewing versus regulating negative emotion induced by highly salient pictures, and whether this pattern related to their self-reported negative affect and suppression success. Despite women reporting more negative affect, both genders had comparable suppression success. Moreover, differences emerged between men and women's FC patterns. During the regulation of negative emotion, better suppression in women was associated with stronger FC within a cingulo-opercular network, while men exhibited stronger FC within posterior regions of the ventral attentional network. We conclude that due to their propensity for higher emotional reactivity, women may employ a frontal top-down control network to downregulate negative emotion, while men may redirect attention away from the aversive stimulus by using posterior regions of the ventral attention network. The findings may have significant implications for understanding women's vulnerability for

33 developing affective disorders and developing targeted individualized treatment.

Keywords: gender differences, emotion regulation, suppression, fMRI, functional 
- Men and women utilize distinct executive control systems to downregulate negative emotion

- Women show stronger connectivity within a cingulo-opercular network during ER

- Men show stronger connectivity within a ventral attention network during ER

- Women utilize top-down control mechanisms in an effort to downregulate negative emotion

- Men utilize attention redirection mechanisms in an effort to downregulate

9 negative emotion 
Gender differences in emotion have been culturally popularized, with the prevailing stereotype being that women are uniformly more emotionally responsive to aversive stimuli than men. While some empirical evidence mirrors the anecdotal conception (Fabes and Martin, 1991; Fischer, 1993; Grossman and Wood, 1993; Hess et al., 2000; Plant et al., 2000; Timmers et al., 2003), some researchers (Garnefski et al., 2004; NolenHoeksema, 2001; Thayer et al., 1994) conjecture the basis for differences in emotion arise due to variations in emotion regulation (ER). Yet, despite the increasing recognition that deficient ER is at the core of various affective disorders (Berking et al., 2014; Dalili et al., 2015; Green et al., 2007; Joormann and Stanton, 2016; O'Driscoll et al., 2014; Phillips et al., 2008), and importantly, that women show an increased prevalence (Kessler et al., 1993; Leach et al., 2008; Nolen-Hoeksema, 2012, 2001; Thomsen et al., 2005), the neural basis of gender differences in ER remains poorly understood.

ER describes processes that individuals use to influence the experience and expression of emotions (Giombini, 2015; Gross, 2015; Ochsner and Gross, 2005). The most prominent neurobiological ER model focuses on the modulatory effect of the prefrontal and cingulate regions involved in top-down control over the affective instantiation regions as a function of one's regulatory goal, tactic, nature of stimuli and emotions being regulated (Ochsner et al., 2012; Ochsner and Gross, 2005). Specifically, brain regions implicated in top-down control include the dorso and ventrolateral, and dorso and ventromedial prefrontal cortex (DLPFC, VLPFC, DMPFC, VMPFC), orbitofrontal cortex (OFC), inferior parietal cortex (IPC) and anterior cingulate cortex (ACC); while those underlying emotion generation comprise the insulae, amygdalae and basal ganglia (Ochsner et al., 2012; Ochsner and Gross, 2005). Within this model

24 however, few (and equivocal) findings have emerged from functional neuroimaging studies investigating gender differences. For example, McRae et al., 2008 showed lower increases in prefrontal activity (ACC, IPC) and greater decreases in amygdala activity during ER efforts in men compared to women, despite no difference in self-reported negative emotion between genders. Domes et al., 2010 found the opposite activation pattern, indicating greater prefrontal activity (VMPFC) in men compared to women during

30 employment of ER, with no notable gender differences in amygdala activity or self-report 31 regulation success. Interestingly, both studies indicate a more efficient ER process in 
1 men, suggesting less effortful cognitive control (McRae et al., 2008) and more precise recruitment of areas putatively underlying ER (Domes et al., 2010) in men compared to women. In parallel, Mak et al., 2009's study argues that while men tend to utilize brain regions underlying cognitive control (lateral PFC and ACC) to a greater extent than women during ER, women tend to use emotion-associated ones (medial OFC), with comparable self-reported regulation success. In an effort to synthesize this contradictory information, a recent meta-analysis assembling the aforementioned studies proposes men may be more efficient in ER as evidenced by greater increases of fronto-parietotemporal activity, and greater decreases of limbic/subcortical activity, while women may experience emotions with greater frequency and intensity as evidenced by increased activity in limbic regions (Whittle et al., 2011).

While some information regarding gender differences in ER may be gleaned from functional activity studies, connectivity within ER-related brain regions may provide 14 another avenue for understanding how men and women differ in regulatory mechanisms. Our previous work has detailed right-hemispheric functional pathways responsible for inhibitory regulation of emotional reactivity between the right anterior middle frontal gyrus 17 (aMFG) and the OFC, and between the right OFC with the amygdala (Depue et al., 2016). We proposed this network may function hierarchically, with higher-order maintenance and updating of task goals performed by the right aMFG, whereas modulation of the amygdala (downstream effectors) implemented by an intermediary region (OFC) that exhibits direct

21 anatomical connectivity. Similar results were found by Banks et al., 2007, a study that showed individuals who are more successful in regulating their emotion demonstrate greater effective connectivity (EC, a measure of connectivity relative to the task) between amygdala and lateral and medial PFC. Yet presently, scant functional connectivity (FC) data between these regions exists examining gender differences related to ER. One such resting-state study found FC within the centromedial amygdala displayed gender-specific variations in association with trait-level ER (Wu et al., 2016). The authors suggest connectivity patterns and higher ER in this sub-region of the amygdala in women was

29 linked to internal and emotional focus, while men's connectivity pattern and higher ER 30 related to a greater ability to downregulate negative emotion compared to women. Lastly,

31 one study investigating gender differences in EC during negative emotion processing 
1 demonstrated EC from the right amygdala to the DMPFC is significantly stronger in men compared to women, with the authors concluding that men may have a more evaluative rather than purely affective, in-the-moment, brain response during negative emotion processing (Lungu et al., 2015). To the best of our knowledge however, no study to date has utilized a network-based approach to examine gender differences in FC during an ER task.

prior studies investigating gender differences during ER, we hypothesized that: (1) women, compared to men, will rate aversive stimuli as more distressing and have

11 increased self-reported negative affect (2) women, compared to men, will show increased 12 connectivity between brain regions putatively underlying emotional response (amygdala, 13 hippocampus) 3) men, compared to women, will show increased connectivity between 14 regions underlying top-down control of emotion (parietal cortex, DLPFC, VLPFC).

\section{METHODS}

\subsection{Participants and Procedure}

17 A total of 55 healthy young adults without a reported history of neurological or psychiatric disorders were recruited for this study (Table 1). An a priori power analysis was conducted using G*Power3 (Faul et al., 2007) to test effects between men and women using a two-tailed test, with a medium effect size based and an alpha of 0.05.

21 Results showed that a total sample of 40 participants with two equal sized groups of $n=20$ was required to achieve a power of .80 . Therefore, we feel confident that our sample size is adequate to detect the desired outcomes. It should be noted, that when compared with

24 the small effect sizes reported in behavioral studies, previous data (Ritchie et al., 2018) suggest that functional brain imaging may provide more sensitive measures of gender differences than purely behavioral measures. All participants were right-handed and had normal or corrected-to-normal vision and hearing. Participants were recruited through oncampus flyers and the Psychology Department's online research participation system (SONA Systems). All participants were paid for their participation. Experimental protocols

30 were approved by University of Louisville's Institutional Review Board prior to data 31 collection, and written informed consent was obtained from each participant prior to 
1 experimental sessions. Seven participants were excluded from analyses due to 2 incomplete behavioral data $(n=1)$, various scanning issues resulting in incomplete fMRI 3 data $(n=4)$, and disclosed psychiatric diagnosis following consent $(n=2)$. This left $48(20$ 4 women) participants in the final sample. Men and women were comparable in age and 5 years of education ( $p=0.69$ and $p=0.90$, respectively).

\begin{tabular}{|ccc|}
\hline \multicolumn{3}{|l|}{ Table 1} \\
\hline \multicolumn{3}{|c|}{ Memographics of Studied Sample } \\
\hline \multicolumn{3}{|c|}{ Women } \\
\hline $\begin{array}{c}\text { Age } \\
\text { (Mean, SD) }\end{array}$ & $21.5(4.1)$ & $22(3.7)$ \\
$\begin{array}{c}\text { Years of Education } \\
\text { (Mean, SD) }\end{array}$ & $14.3(2.43)$ & $14.3(2.4)$ \\
\hline
\end{tabular}

6 After careful screening, participants signed a consent form, were briefed on fMRI protocol, 7 and received the Emotion Regulation Task (ERT) instructions. Stimuli were displayed 8 using ePrime onto an Invivo Esys LCD TV monitor at the back of the scanner bore, which 9 was viewed by participants through a mirror on the head-coil.

\section{2fMRI Task - Emotion Regulation Task (ERT)}

The ERT was divided into three parts: (A) ER Baseline (B) Emotion Regulation (ER)

12 (C) ER Rating. Only parts A \& B were BOLD scans, part C was structural.

ER Baseline:

14 The ER Baseline task employed an event-related design, during which 20 aversive 15 International Affective Picture System (IAPS) pictures and 10 neutral pictures were 16 displayed (Lang et al., 2005). The pictures were displayed for 4 seconds in a pseudo17 random order. The pseudorandom order is utilized in order to achieve a maximum 18 additive effect of the elicited hemodynamic response. Following presentation of each 19 stimulus, participants rated the image for how negative it made them feel, using a 4-point 20 Likert scale $(1=$ None to $4=$ Extremely negative; 2 seconds to rate; Figure 1$)$. This 21 allowed acquisition of a subjective negative baseline rating for each participant. A 
1 pseudorandom variable jittered ITI was incorporated to increase design efficiency for hemodynamic response estimation $(0-10 \mathrm{sec})$. Resultant behavioral ratings from the task were further used to calculate ER Suppression Score (see part C).

(B) Emotion Regulation (ER):

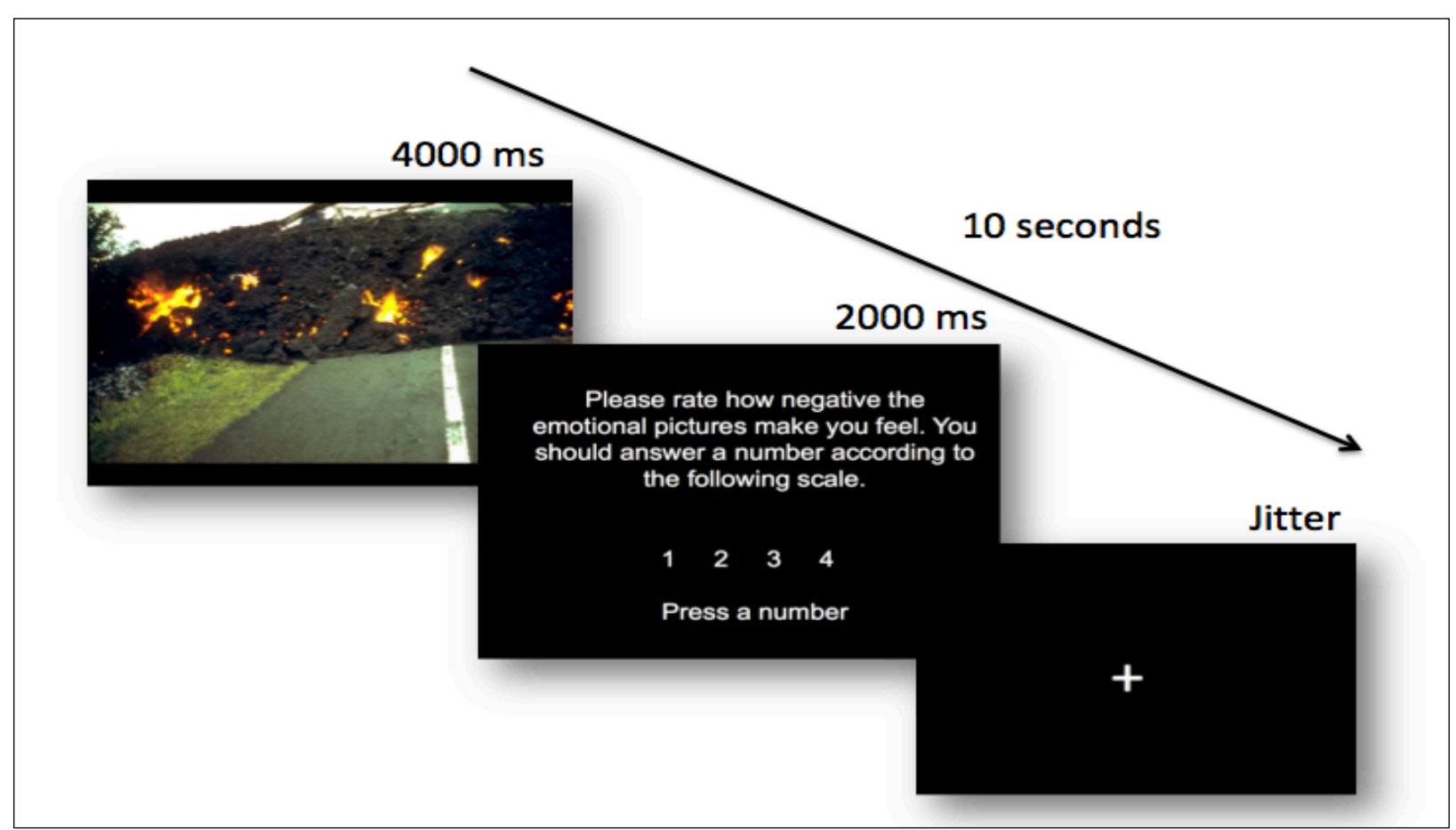

The ER task also employed an event-related design, whereby a different set of aversive and neutral pictures selected from the IAPS (Lang et al., 2005) were displayed. First, the

9 words 'SUPPRESS' in the color red or 'VIEW' in the color blue appeared as cues for 500

10 ms to prepare the participant for the upcoming picture. Next, negative pictures surrounded

11 by a red border (Suppress trials) and neutral pictures surrounded by a blue border (View

12 trials) were presented for 3.5 seconds in a pseudo-random order (Suppress trials $n=30$,

13 View trials $n=12$ ) (Figure 2). The participant was instructed to "decrease or detach' from

14 the emotion when the border was red, and 'simply view' the picture when the border was

15 blue". The "Suppress" instructions were worded simply in order to encourage the

16 participant to use their default ER method. A pseudorandom variable jittered ITI was

17 incorporated to increase design efficiency for hemodynamic response estimation (0-4

$18 \mathrm{sec})$. The pictures repeated once, with a 60 second break in-between. To test the

19 successful induction of negative emotion during the ER Task, a two-tailed paired samples 
$1 t$-test was conducted between mean ratings of the View $(M=1.3, S D=0.52)$ and Suppress $2(M=2.5, S D=0.52)$ pictures, which revealed a significant difference between the two 3 conditions $t(47)=-10.59, p<0.01$.

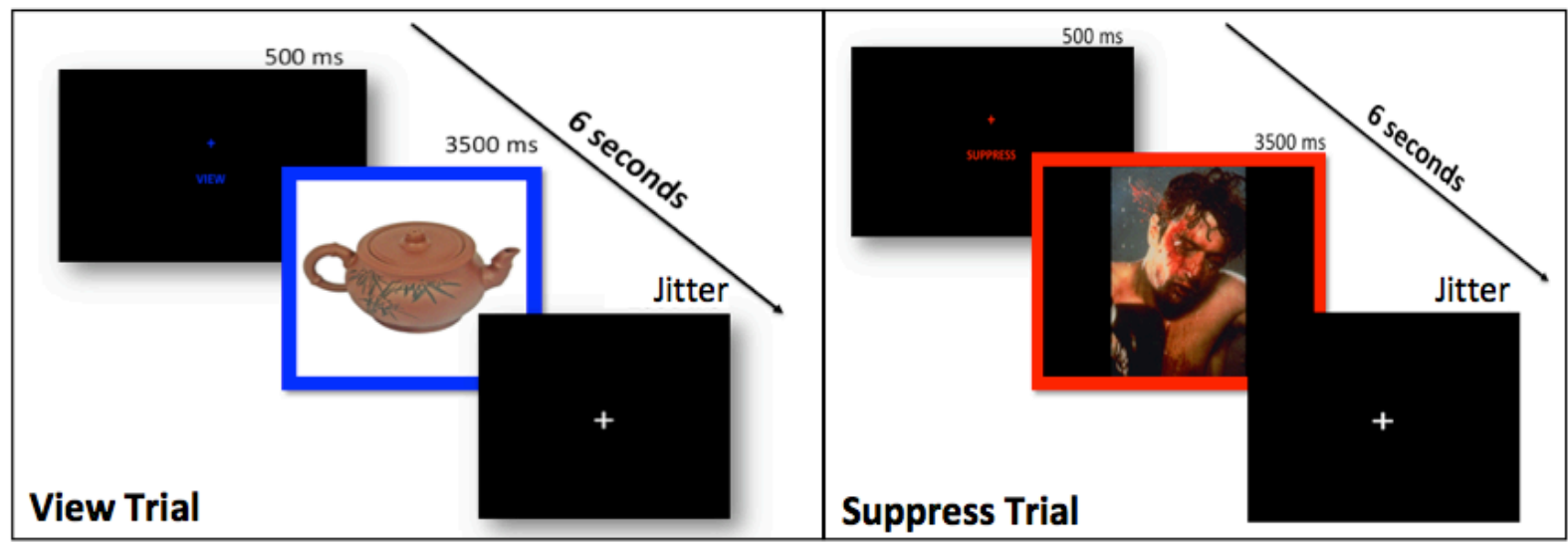

Figure 2: Example of a single trial of each condition from Emotion Regulation (ER).

(C) ER Rating

During a structural scan, the same images used in part B were then displayed without any border for 4 seconds. Following presentation of each image, participants rated how negative the image made them feel, using a four-point Likert scale $(1=$ None to $4=$ Extremely negative, displayed in the same way as ER Baseline). To test whether the participants were successfully able to down-regulate their emotional response to the images, a two-tailed paired samples $t$-test was conducted between the negative Baseline Ratings $(M=2.7, S D=0.50)$ and $E R$ negative ratings $(M=2.4, S D=0.52)$, showing a significant difference in scores $t(46)=5.4, p<0.01$. Furthermore, the negative ER ratings from this part were subtracted from the earlier acquired negative baseline ratings (part $A$ ) in order to calculate a Suppression Score. The Suppression Score was derived from negative ratings only (rather than ratings on "View" trials) to ensure an accurate measurement of negative affect down-regulation success (i.e. comparing a response to negative stimuli before and after ER). The Suppression Score was used as an independent variable in subsequent FC analyses (dependent variable).

\subsection{Imaging Data Acquisition}

\subsubsection{Structural}


All structural MRI images were acquired using a Siemens 3-T Skyra MR scanner. A 20-channel head coil was used for radiofrequency reception. Participants were given earplugs to reduce scanner noise and were additionally given headphones to receive instructions. Foam padding was added to limit motion if additional room remained within the head coil, and a piece of folded tape was placed over the participant's forehead as a reminder to remain still throughout the scan. Structural images were obtained via a T1weighted magnetization-prepared rapid gradient-echo sequence (MPRAGE) in 208 sagittal slices. Imaging parameters were as follows: echo time $(T E)=2.26 \mathrm{~ms}$, repetition time $(\mathrm{TR})=1700 \mathrm{~ms}$, flip angle $=9.0^{\circ}$, field of view $(\mathrm{FoV})=204 \mathrm{~mm}$, and voxel size $=0.8$

11 with this study.

\section{$12 \quad 2.4 .2$ Functional}

13 Functional blood oxygenation level-dependent (BOLD) images were collected using 14 gradient-echo $2^{*}$-weighted echoplanar imaging (TE = $28 \mathrm{~ms}$; TR $=2000 \mathrm{~ms}$; flip angle $15=79^{\circ} ; \mathrm{FoV}=204 \mathrm{~mm}$; voxel size $=3.2 \mathrm{~mm}^{3}, 38$ interleaved slices). Slices were oriented 16 obliquely along the AC-PC line.

\section{$17 \quad 2.4$ Imaging Data Analysis}

All analyses and visualizations were conducted using the CONN toolbox 18.b (Whitfield-Gabrieli and Nieto-Castanon, 2012) based on SPM12 (Penny et al., 2007) in the 2017 version of MATLAB. Spatial preprocessing in the CONN toolbox included realignment, normalization and smoothing ( $8 \mathrm{~mm}$ FWHM Gaussian filter) using SPM12 default parameter settings. Anatomical volumes were segmented into gray matter, white matter and cerebrospinal fluid (CSF) areas, and the resulting masks were eroded to minimize partial volume effects. The temporal time series characterizing the estimated subject motion, as well as the blood-oxygen-level dependent (BOLD) fMRI time series within the subject-specific white matter mask (five principal component analysis (PCA) parameters) and the CSF mask (five PCA parameters), were used as temporal covariates and removed from the BOLD functional data using linear regression. Stimuli onsets and duration were specified in the toolbox, so that BOLD time series could be appropriately

30 divided into task-specific blocks. Block regressors were then convolved with a canonical

31 hemodynamic response function, and subsequently temporally filtered. The resulting 
1 residual BOLD time series were then band-pass filtered $(0.008-\mathrm{inf} \mathrm{Hz})$, as this filter

2 benefits from keeping higher-frequency information fitting event-related tasks (Nieto-

3 Castanon, 2015a).

4 A region of interest ( $\mathrm{ROI}$ ) based correlation approach was used to evaluate temporally

5 correlated BOLD signal between 28 a priori selected 10mm ROls from CONN's atlas

6 (Harvard-Oxford). The selection of these ROls was based on previous research

7 demonstrating their involvement in ER (Depue et al., 2016; Kohn et al., 2014; Picó-Pérez

8 et al., 2017). They included: bilateral - ventral frontal pole (FP), orbital frontal cortex

9 (OFC), IFG operculum (IFG oper), middle frontal gyrus (MidFG), inferior frontal gyrus

10 (IFG, pars opercularis), inferior frontal gyrus (IFG, pars triangularis), posterior

11 supramarginal gyrus (pSMG), angular gyrus (AG), dorsal anterior cingulate gyrus (dACC),

12 anterior parahippocampal gyrus (aPaHC), posterior parahippocampal gyrus ( $\mathrm{pPaHC}$ ),

13 hippocampus, amygdala, and the subcallosal Cortex (SubCalc), medial dorsal anterior

14 cingulate gyrus (dACC) (Table 2). 
Table 2

Selected Regions of Interest (ROIs) for Connectivity Matrices

ROI Abbreviation ROI Name

$\begin{array}{lllrr}\text { R FP } & \text { Right Ventral Frontal Pole } & 26.15 & 52.14 & -8.25 \\ \text { L FP } & \text { Left Ventral Frontal Pole } & -24.72 & 52.95 & 7.5 \\ \text { R OFC } & \text { Right Orbital Frontal Cortex } & 29.11 & 23.07 & -16.23 \\ \text { L OFC } & \text { Left Orbital Frontal Cortex } & -29.54 & 23.66 & -16.57 \\ \text { R IFG Oper } & \text { Right IFG, Operculum Cortex } & 41.11 & 18.62 & 4.91 \\ \text { L IFG Oper } & \text { Left IFG, Operculum Cortex } & -39.7 & 18.32 & 4.52 \\ \text { R MidFG } & \text { Right Middle Frontal Gyrus } & 39.11 & 18.62 & 42.78 \\ \text { L MidFG } & \text { Left Middle Frontal Gyrus } & -38.07 & 18.43 & 42.06 \\ \text { R IFG tri } & \text { Right IFG, pars triangularis } & 51.86 & 27.76 & 7.7 \\ \text { L IFG tri } & \text { Left IFG, pars triangularis } & -49.71 & 28.49 & 8.66 \\ \text { R IFG oper } & \text { Right IFG, pars opercularis } & 52.21 & 15.41 & 16.2 \\ \text { L IFG oper } & \text { Left IFG, pars opercularis } & -50.64 & 14.51 & 15.39 \\ & \text { Right Supramarginal Gyrus, } & & & \\ \text { R pSMG } & \text { posterior division } & 55.2 & -40.36 & 33.6 \\ & \text { Left Supramarginal Gyrus, posterior } & & \\ \text { L pSMG } & \text { division } & -54.88 & -46.02 & 33.24 \\ \text { R AG } & \text { Right Angular Gyrus } & 51.93 & -51.8 & 32.35 \\ \text { L AG } & \text { Left Angular Gyrus } & -50.35 & -55.7 & 29.76 \\ & \text { Dorsal Anterior Cingulate Gyrus } & & & \\ \text { dACC } & \text { (medial) } & 0.8 & 18.29 & 24.34 \\ \text { R dACC } & \text { Right Anterior Dorsal Cingulate } & & & \\ \text { L dACC } & \text { Gyrus } & 6.55 & 36.56 & 22.69 \\ & \text { Left Anterior Dorsal Cingulate } & & & \\ \text { R aPaHC } & \text { Gyrus } & -6.2 & 36.65 & 20.78 \\ \text { L aPaHC } & \text { Right Parahippocampal Gyrus, } & & & \\ \text { R pPaHC } & \text { anterior division } & 22.35 & -8.05 & -30.25 \\ & \text { Left ParahippocampalGyrus, } & & & \\ \text { L pPaHC } & \text { anterior division } & -21.86 & -9.1 & -30.3 \\ \text { R Hip } & \text { Right Parahippocampal Gyrus, } & & & \\ \text { L Hip } & \text { posterior division } & 22.9 & -30.53 & -16.75 \\ \text { R Amy } & \text { Left Parahippocampal Gyrus, } & & & \\ \text { L Amy } & \text { posterior division } & -21.89 & -32.42 & -16.88 \\ \text { SubCalc } & \text { Right Hippocampus } & 26.49 & -20.95 & -14.25 \\ & \text { Left Hippocampus } & -25.17 & -23.19 & -13.8 \\ & \text { R Amygdala } & 23.08 & -3.98 & -17.68 \\ \text { L Amygdala } & -22.99 & -4.94 & -17.73 \\ & \text { SubcallosalCortex } & -0.07 & 20.53 & -14.83\end{array}$


The CONN toolbox was used to obtain a linear measure of FC based on bivariate correlation and regression coefficients, as well as their associated multivariate measures of semi-partial correlation and multivariate regression coefficients (Whitfield-Gabrieli and 4 Nieto-Castanon, 2012). Wilks' lambda or F-statistics were used to evaluate ROI-to-ROI 5 connectivity matrices for each participant depending on the dimensionality of the between-subjects' contrasts (ER Baseline: Negative, Negative vs. Neutral; ER Task: Suppress, Suppress vs. View). Effect sizes for connectivity contrasts between all ROI sources were calculated alongside $T$, and $F$ values; and false discovery rate-corrected (FDR) $p$-values were calculated for each specified second-level analysis. The T-stat represents the results of between-subjects conditions contrasts when testing each individual connection separately between each pair of seed and target ROIs, while the Fstat represents the same results but testing each individual seed region separately and looking for any effects across all target ROls (Nieto-Castanon, 2015b). We were interested which particular 28 a priori ROls show differences in FC between genders, therefore we report T-stats and FDR-corrected ( $p$-value of 0.05 ) (seed-level) results below. The CONN toolbox ROI-to-ROI analyses results are considered appropriately corrected for multiple comparisons across all brain and analysis voxels when the voxellevel and the extent cluster-level thresholds use an analysis-wise false positive control FDR-corrected p-values method (Whitfield-Gabrieli and Nieto-Castanon, 2012).

\section{RESULTS}

\subsection{Behavioral Data}

To examine subjective emotion ratings prior to emotion regulation (ER Baseline; part A), we conducted an independent samples $t$-test comparing the means of self-reported negative affect between genders (based on 5000 bootstrap samples). The results showed a statistically significant difference between men's $(M=2.57, S D=0.54)$ and women's $(M=2.99, S D=0.33)$ negative ER Baseline ratings, $t(45)=-3.04, p=0.004,95 \% \mathrm{Cl}[-0.70$ to $-0.14]$, but not between their neutral ER baseline ratings $t(45)=-1.35, p=0.18,95 \% \mathrm{Cl}[-$ 0.21 to 0.04 ] (Figure 3). 


\section{ER Baseline Ratings}

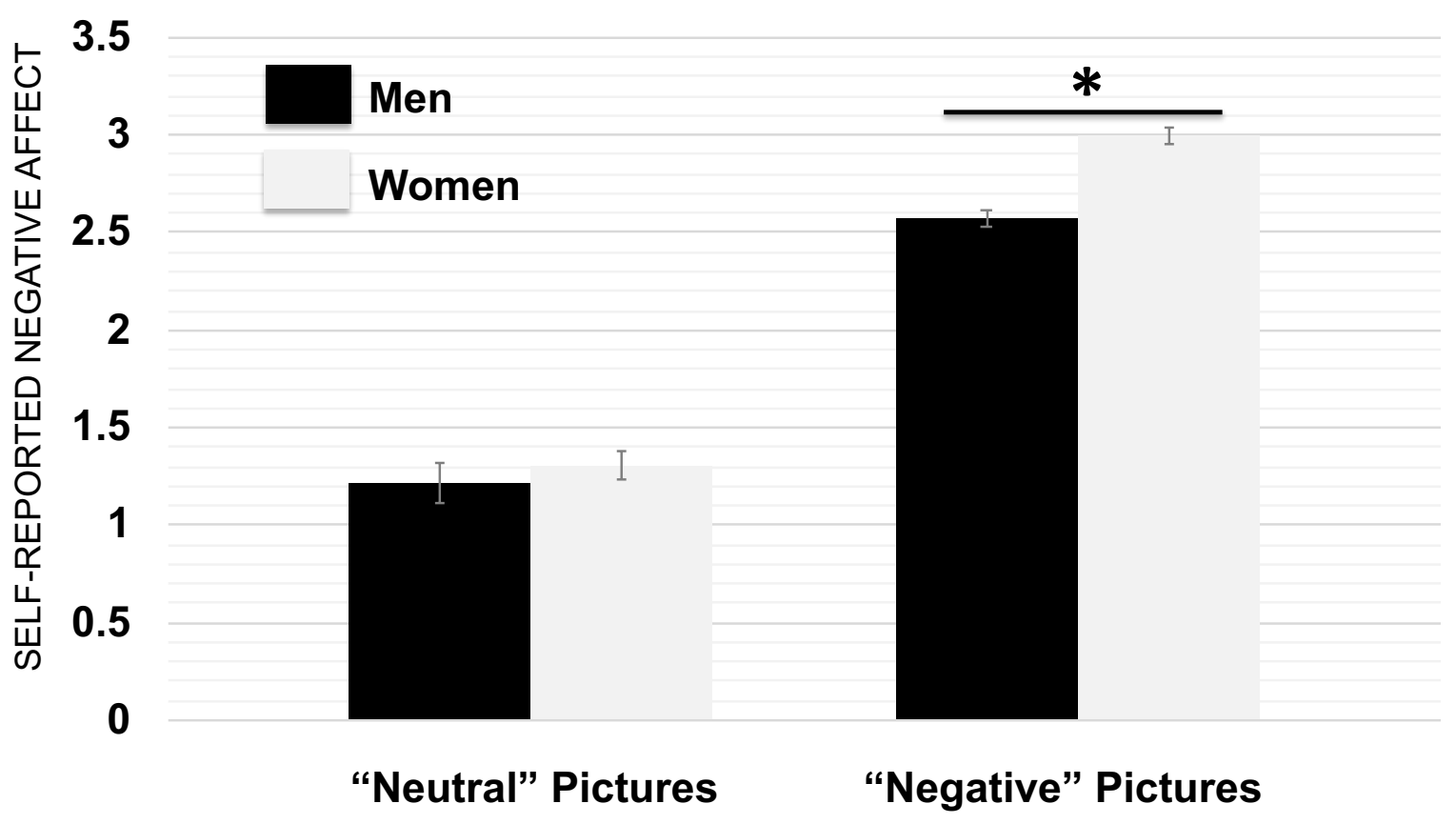

Figure 3: Gender differences in ratings of self-reported negative affect during the ER Baseline Task (part A). Each subject was asked to look and respond naturally to neutral (Neutral) and negative pictures (Negative). Error bars represent standard error the mean (SEM), ${ }^{*}$ represents $p<0.000001$.

To examine subjective emotion ratings during emotion regulation (ER Task; part B),

2 as well as any gender differences in the ability to down-regulate negative emotion 3 (Suppression Score), we conducted an independent samples t-test comparing the means 4 of self-reported negative affect between genders (based on 5000 bootstrap samples).

5 The results showed a statistically significant difference between men's ( $M=2.29, S D=.52)$

6 and women's $(M=2.64, S D=.47)$ negative ER ratings $t(45)=-0.35, p=0.02,95 \% \mathrm{Cl}[-.65$

7 to -.06$]$, but not between their neutral ER ratings $t(45)=-.75, p=0.45,95 \% \mathrm{Cl}[-0.45$ to 0.16$]$

8 (Figure 4) or their Suppression Score $t(45)=-.58, p=0.56,95 \% \mathrm{Cl}[-0.30$ to 0.17$]$ (Figure 9 5). 


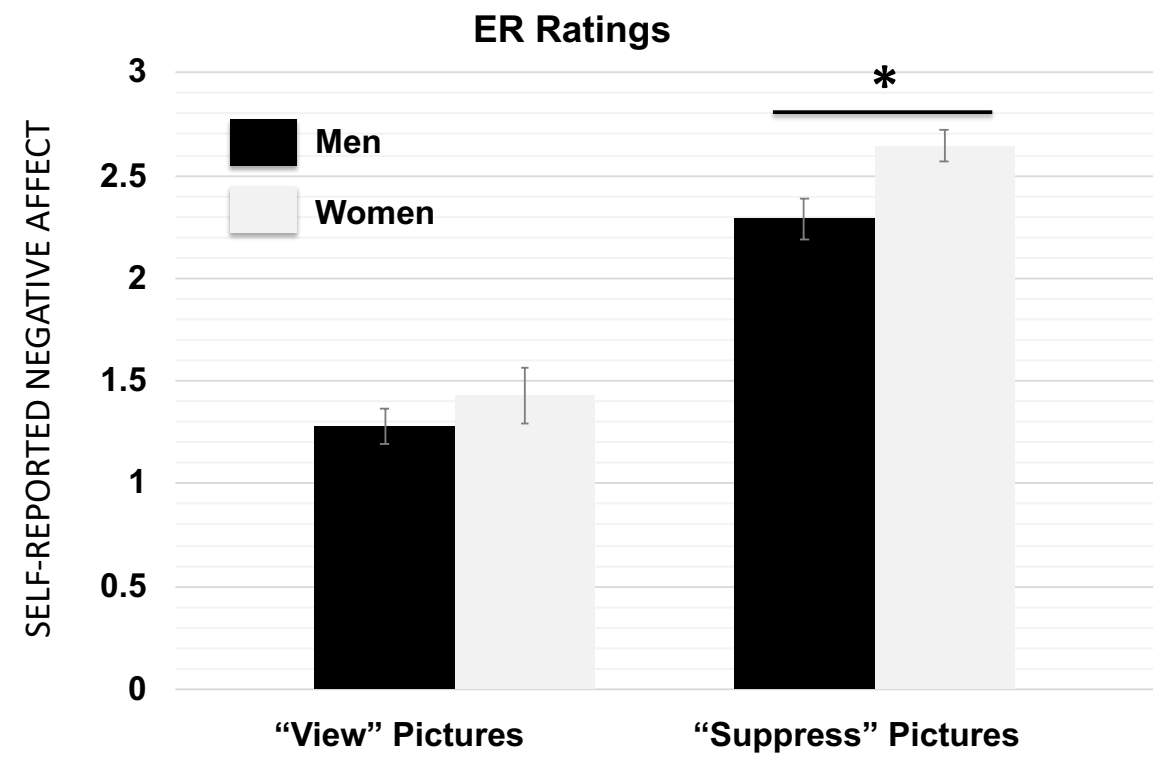

Figure 4: Gender differences in ratings of self-reported negative affect during the ER Task (part B). Each subject was asked to "simply view" the neutral pictures ("View" Pictures) and to "decrease or detach from the emotion" for negative pictures ("Suppress" Pictures). Error bars represent standard of the mean (SEM), * represents $p<0.05$.

Negative Emotion Down-Regulation Success

0.5

0.45

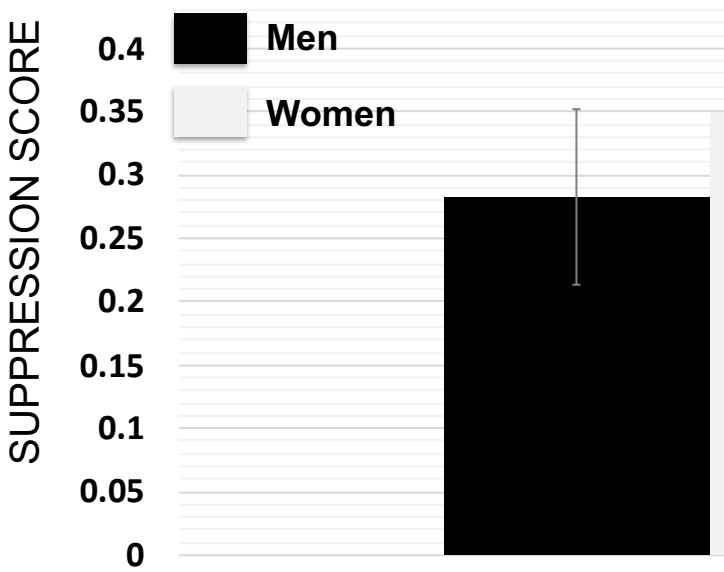

Figure 5: Gender differences in negative emotion down-regulation success (Suppression Score). The Suppression Score was derived from subtracting negative ER ratings (part C) from the earlier acquired negative baseline ratings (part A). Error bars represent standard of the mean (SEM). 


\subsection{Neuroimaging Results}

2 3.2.1 Gender Differences in Functional Connectivity during ER Baseline (part A)

3 To examine whether men and women exhibited neurobiological differences when viewing

4 negative stimuli, we tested the ER Baseline (part A) prior to emotion regulation. As shown

5 in Figure 6, Table 3) below, during the Negative Condition of the ER Baseline, compared

6 to men, women showed increased FC between L FP, L Hip and L pPaHC, and between

7 the R Hip and R AG.

\section{ER Baseline Women > Men Negative Condition}

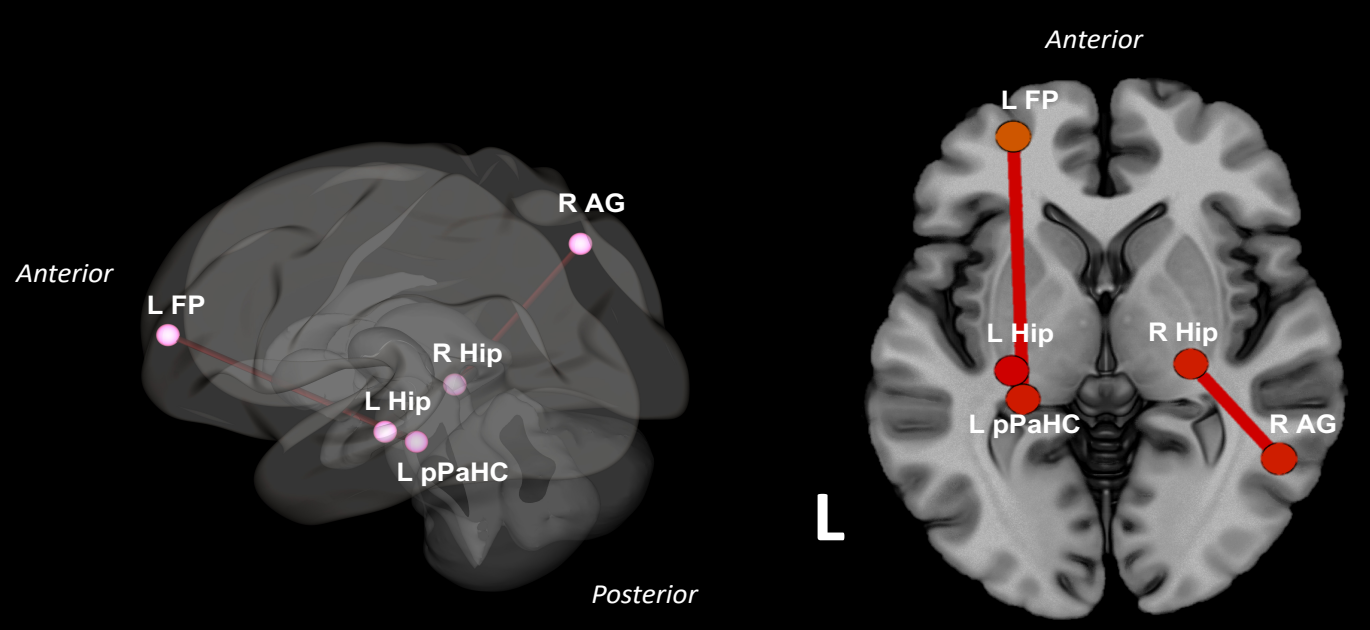

Posterior

Figure 6: Gender differences in functional connectivity during the negative condition of $E R$ Baseline.

Table 3

\begin{tabular}{lllll}
\hline ER Baseline & & & & \\
\hline ROI & ROI & T & p-FDR & Effect Size \\
L pPaHC & L Hip & -3.31 & 0.04 & 0.19 \\
L pPaHC & L FP & -3.24 & 0.04 & 0.14 \\
R Hip & R AG & -3.35 & 0.04 & 0.17
\end{tabular}




\subsubsection{Gender Differences in Functional Connectivity during ER Task (part B)}

2 To examine whether men and women exhibited FC differences during emotion regulation

3 (ER Task; part B), we examined gender differences with respect to suppression, as well 4 as the correlation of FC during the ER Task with self-reported negative affect (ER Ratings, 5 (part C)), and Suppression Score (ER Negative Baseline Ratings - Negative ER Ratings).

$6 \quad$ 3.2.2.1 ER task

7 As shown in Figure 7, Table 4, women, during the Suppress Condition of the ER Task, 8 compared to men, showed increased FC between L IFG Oper and R pPaHC, between R 9 IFG Oper and L pPaHC, and between L Hip and L pPaHC. In addition, women showed 10 decreased connectivity between L pSMG, R pSMG and R AG.

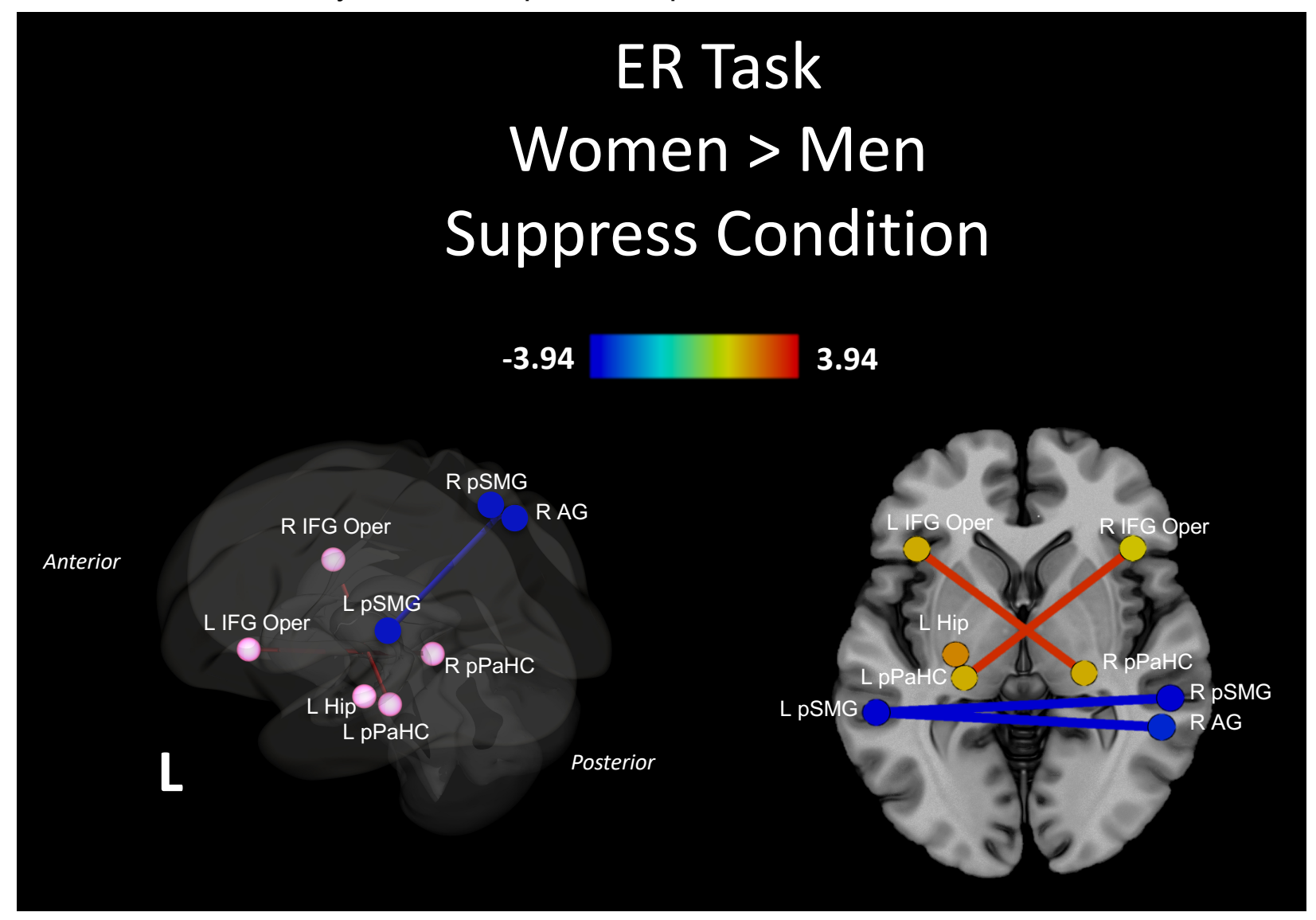

Figure 7: Gender differences in functional connectivity during the suppress condition of the ER Task. 
Table 4

\begin{tabular}{lllll}
\hline ER Task & & & & \\
\hline ROI & ROI & T & p-FDR & Effect Size \\
R AG & L pSMG & -3.94 & 0.007 & 0.27 \\
L pSMG & R pSMG & -3.86 & 0.009 & 0.31 \\
R pPaHC & L IFG Oper & 3.33 & 0.04 & 0.07 \\
L pPaHC & R IFG Oper & 3.38 & 0.02 & 0.12 \\
L pPaHC & L Hip & 3.26 & 0.02 & 0.18
\end{tabular}

$1 \quad$ 3.2.2.2 ER Task Regressed with Self-Reported Negative Affect

2 When assessing the relationship between ER Task FC and self-reported negative affect

3 (negative ratings from ER Rating Task (part C)), women's decreased self-reported

4 negative affect, compared to men's, related to increased FC between R pSMG and L IFG

5 Oper, and between R pSMG and R IFG Oper, L OFC and dACC during suppression

6 (Figure 8, Table 5). 


\section{ER Task}

\section{Regression with Self-Reported Negative Affect Women > Men Suppress Condition}

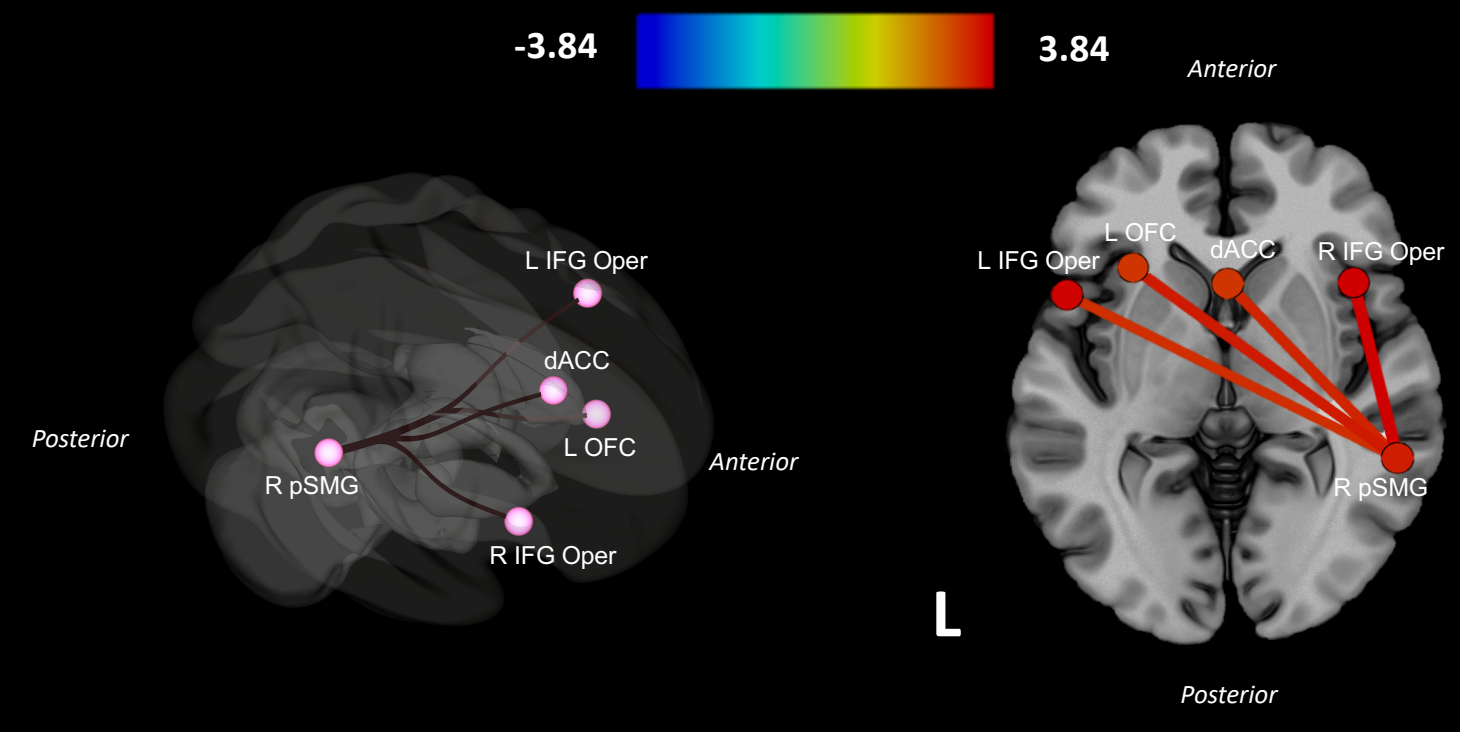

Figure 8: Gender differences in functional connectivity during the suppress condition of the ER Task, regressed with self-reported negative affect.

Table 5

\begin{tabular}{lllll}
\hline \multicolumn{5}{l}{ ER Task Connectivity with Self-Reported Negative Affect } \\
\hline ROI & ROI & T & p-FDR & Effect Size \\
R pSMG & L IFG Oper & 3.24 & 0.01 & 0.41 \\
R pSMG & R IFG Oper & 3.84 & 0.01 & 0.43 \\
R pSMG & L OFC & 3.49 & 0.01 & 0.35 \\
R pSMG & dACC & 3.36 & 0.01 & 0.35
\end{tabular}




\section{$1 \quad$ 3.2.2.3 ER Task Regressed with Suppression Score}

2 Lastly, we assessed the relationship between ER Task FC and Suppression Score (ER

3 negative ratings (part C) subtracted from ER negative baseline ratings). In the Suppress

4 contrast, increased Suppression Score (better suppression) in women, compared to men,

5 was associated with increased FC between L IFG tri and R IFG Oper, and between L IFG

6 tri and R dACC (Figure 9, Table 6).

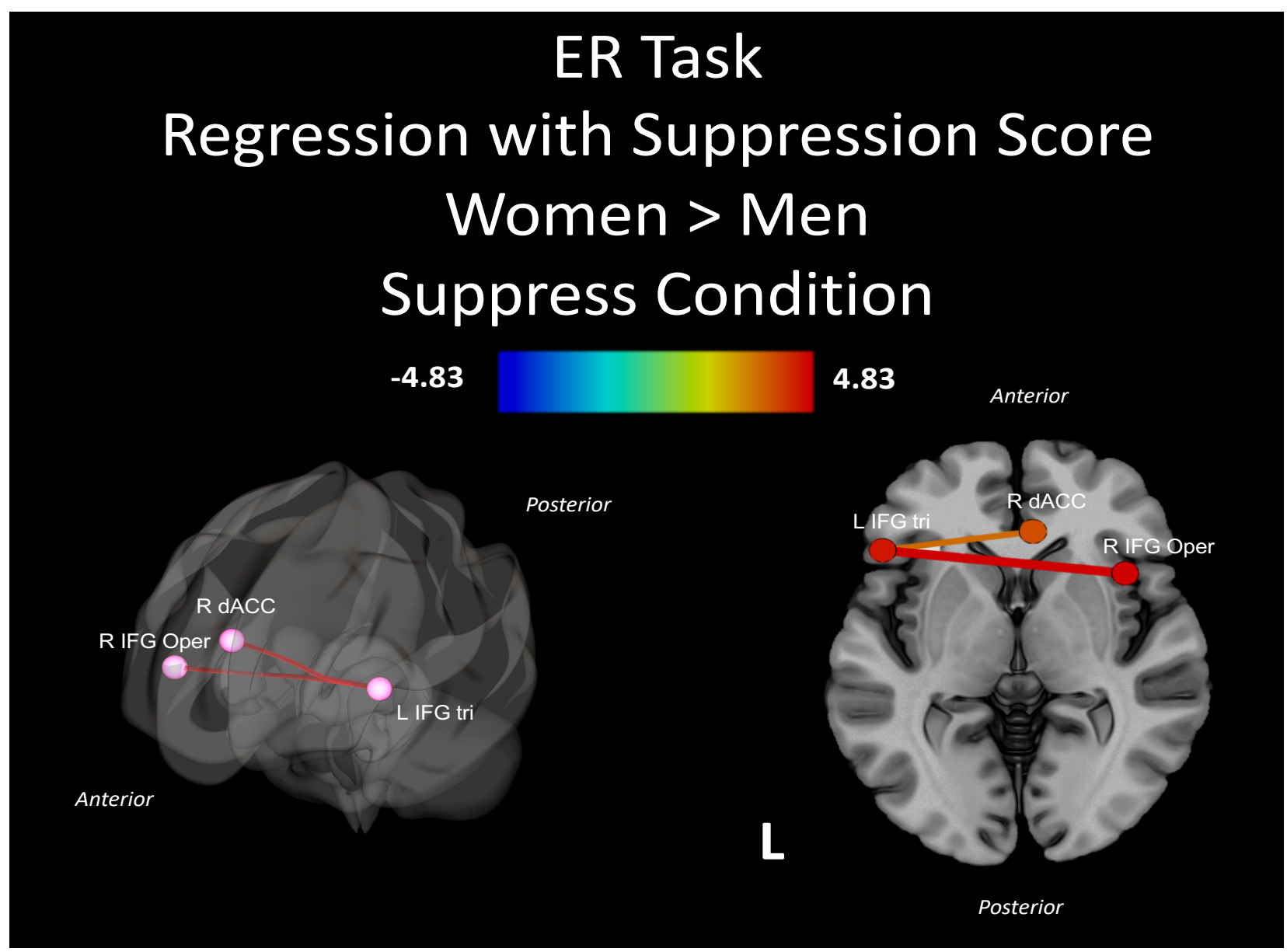

Figure 9: Women vs Men's FC during the ER task, Suppress Condition, regressed with Suppression Score.

Table 6

ER Task Connectivity with Suppression Score

\begin{tabular}{lllll}
\hline ROI & ROI & T & p-FDR & Effect Size \\
L IFG tri & R IFG Oper & 4.83 & 0.0005 & 0.63 \\
L IFG tri & R dACC & 3.18 & 0.0300 & 0.47
\end{tabular}




\section{DISCUSSION}

The present fMRI study investigated whether gender differences existed in FC between brain regions underlying regulation of negative emotion during an ER task. Behaviorally, we observed that despite women rating the negative stimuli as more distressing compared to men, they had comparable Suppression Scores. However, the ER task revealed gender differences in FC. Specifically, in women, enhanced ability to down-regulate negative emotion related to increased recruitment of a cingulo-opercular network, and in contrast, posterior regions of a ventral attention network in men. Altogether, our results suggest men and women may utilize distinct executive control neural mechanisms in the control of negative emotion. To our knowledge, this is the first study to explore FC differences between men and women while engaging in ER and relate those differences to self-reported negative affect and suppression ability.

Women self-reported having more negative affect than men across tasks as we predicted, yet this difference disappeared in the process of down-regulating negative emotion. Our results are consistent with previous findings showing women are more expressive (Hampson et al., 2006; Kring and Gordon, 1998), experientially reactive (Gard and Kring, 2007) and sensitive (Li et al., 2008) to aversive stimuli. Moreover, our results mirror the Domes et al., 2010 and McRae et al., 2008 observations that, despite their heightened emotional reactivity, women had comparable suppression scores compared to men. This disassociation implies that there could be gender-related differences in the pattern of FC associated with ER.

We observed that while viewing negative stimuli (ER Baseline task), women exhibited increased FC between the left ventral FP, hippocampus and $\mathrm{pPaHC}$, as well as between 24 right $A G$ and hippocampus compared to men. Connectivity between ventral FP and hippocampus has been consistently associated with the recollection memory network (Adnan et al., 2016; Greenberg et al., 2005) and memory-based decision making (Weilbächer and Gluth, 2017). In regard to the pPaHC, this structure has been shown to be a key node of the recollection memory network, which together with lateral parietal cortices are preferentially engaged when individuals recall rich contextual details (Adnan 30 et al., 2016), particularly about novel scenes (Howard et al., 2013). Furthermore, the AG

31 is part of a largely right-lateralized, well validated ventral attention system specialized for 
1 the detection of behaviorally relevant stimuli (Corbetta and Shulman, 2002). Notably, research suggests women are more likely to direct attention towards a novel item compared to men (Brown, 2013; Stoet, 2010). Together, our FC findings suggest that compared to men, women may engage a bottom-up emotional memory processing network when simply viewing negative stimuli, likely due to the allocation of attention to the highly salient stimuli.

During the regulation of negative emotion (ER Task), we observed women exhibited increased FC between the bilateral IFG and hippocampus and $\mathrm{pPaHC}$, while men exhibited increased FC between the AG and bilateral PSMG. The IFG has been well established as a crucial brain region recruited during the volitional inhibition of affective response (Aron et al., 2014; Konishi et al., 1999; Ochsner et al., 2012), as well as pivotal in coordinating encoding processes in conjunction with the hippocampus (Addis and McAndrews, 2006). The AG and pSMG, regions which underlie attention re-allocation to behaviorally salient stimuli (Corbetta and Shulman, 2002), have previously been associated with a more automatic ER process (Viviani, 2013). Furthermore, a recent meta-analysis suggested the ER process may be explained not only as frontal regions inhibiting limbic regions, but also those underlying attention (Frank et al., 2014). In contrast to previous findings arguing men may utilize brain regions underlying top-down cognitive control to a greater extent than women during ER (Mak et al., 2009), our results suggest that women employ a bilateral top-down cingulo-opercular control network in order to suppress negative emotion. Regarding the FC findings in men, ER theorists have suggested the existence of an automatic (Mauss et al., 2007; Phillips et al., 2008), or an unconscious variety of emotion regulation (Bargh and Williams, 2007) which contrasts with the deliberate voluntary form typically interrogated (Ochsner et al., 2012; Ochsner and Gross, 2005) and relies on the attentional reorienting mechanisms located in the ventral attention network (Viviani, 2013). Therefore, we speculate women may utilize a more deliberate ER process reliant on frontal regions, while men may employ a more efficient or automatic ER process dependent on regions within the posterior regions of the ventral attention network.

In parallel, we found decreased self-reported negative affect in women (compared to men) related to increased connectivity between right PSMG and left IFG, dACC and OFC. 
1 The $\mathrm{dACC}$ lies in a unique anatomical position, with connections to both the limbic system and PFC, and has an important role in integrating neuronal circuitry for affect regulation (Stevens et al., 2011). Moreover, the dACC plays a crucial role in conflict monitoring (Lau et al., 2006; Stuss and Levine, 2002; Thomsen et al., 2005) as well as integration of attentional and emotional stimuli (Yamasaki et al., 2002). Hence, dACC connectivity with frontal and parietal structures in our results may point to a deliberate effort on the part of women to resolve the heightened emotional conflict during the ER process. Similarly, previous studies implicate the OFC in top-down modulation of autonomic responses to emotional experiences (Ohira et al., 2006; Phillips et al., 2003), and it appears larger in women than men (Gur, 2002). Therefore, our findings suggest decreased self-reported negative affect in women may relate to the recruitment of a fronto-parietal top-down control network in order to modulate the autonomic physiological responses evoked by the highly salient emotional stimuli.

Lastly, we observed that in women (compared to men), better suppression of negative emotion related to increased connectivity between bilateral IFG and R dACC. Along with the IFG, robust evidence exists for the recruitment of the dACC during the ER process as

17 a conflict monitoring tool (Mak et al., 2009; McRae et al., 2008; Ochsner et al., 2012;

18 Ochsner and Gross, 2005; Weissman et al., 2005). That is, the dACC may underlie monitoring cognitive conflict and recruits other prefrontal regions to resolve this conflict when necessary (Botvinick et al., 2004; Fan et al., 2003), putatively in order to reduce amygdala activity (Etkin et al., 2006). Previous studies investigating gender differences in ER suggest women tend to utilize emotion-associated brain areas, while men tend to utilize cognitive ones (Kohn et al., 2014; Mak et al., 2009), and even that men may possess a greater ability to regulate their emotions compared to women (Kong et al., 2014). In contrast, our novel FC findings demonstrate that women downregulate negative emotion as successfully as men by recruiting a cingulo-opercular network.

To our knowledge, these results provide the first FC evidence that indicate women suppress negative emotion as well as men by exhibiting a cingulo-opercular network,

29 while men exhibit a posterior region of the ventral attentional network. Since women 30 reported increased negative affect compared to men, we suggest that the heightened 31 emotional response required utilization of the top-down cingulo-opercular control network 
1 to effectively downregulate the negative emotion. Men recruited posterior regions of the 2 ventral attentional network while suppressing negative emotion, perhaps due to finding 3 the negative stimuli less aversive as women, and conceivably requiring an efficient and 4 automatic form of ER. The FC findings contribute an alternate avenue for investigating gender differences within the neural correlates of ER, which may explain women's greater prevalence of developing affective disorders, particularly depression and anxiety (Kessler et al., 1993; Leach et al., 2008; Nolen-Hoeksema, 2012, 2001; Thomsen et al., 2005). Indeed, research suggests that in major depressive disorder, functional and structural abnormalities within cingulo-opercular pathways could contribute to maladaptive forms of self-focused processing and rumination (Pizzagalli, 2011).

11 Two limitations in our study can be noted. Firstly, the lack of control of hormonal cycle 12 or contraception method for female participants poses a problem. Indeed, it has been 13 shown that social processes, and in particular the neural response to emotion regulation, 14 may vary as a function of hormonal phase of the women (Dan et al., 2019). Secondly, subjective negative affect ratings may not be the most valid measures to reflect emotional experiences, and that the inclusion of physiological responses to emotion could have provided a more reliable index (Ohira et al., 2006).

In conclusion, the present study demonstrated gender-specific FC patterns associated with negative emotion suppression during an ER task, which corresponded with selfreported negative affect and its downregulation success in a group of healthy individuals.

21 Specifically, the results indicated women utilize a cingulo-opercular network to downregulate negative emotion, while men employ posterior regions of the ventral attention network, with comparable suppression outcomes. We speculate the dissociation in the use of executive control mechanisms is likely due to increased emotional reactivity commonly observed among women and an enhanced need to resolve heightened emotional conflict. The findings may provide a better understanding of the mechanisms underlying gender differences with respect to ER. Enhanced knowledge of genderspecific neural bases of ER may help illuminate women's vulnerability to affective disorders and critically, may facilitate the design of personalized therapeutic interventions,

30 for instance utilizing neurofeedback (Linhartová et al., 2019), to target gender-specific 31 neural networks underlying ER. 


\section{Acknowledgements}

2 Many thanks to Dr. Nick Hindy, Dr. Ali Khatibi, and Dr. Andrea Olinger for invaluable

3 advice regarding writing.

5 Data availability

6 Raw data and behavioral variables of interest for the empirical results presented here can

7 be found on the Mendeley Data Repository.

9 Declarations of Interest

10 None

11

12 CRediT Author Statement

13

14 Teodora Stoica: Data Curation, Investigation, Project administration, Formal analysis,

15 Methodology, Visualization, Writing - original draft Lindsay Knight: Project

16 administration Farah Naaz: Conceptualization, Project administration, Investigation,

17 Data Curation Samantha Patton: Writing - Editing Brendan Depue: Resources,

18 Conceptualization, Supervision, Writing - Review and editing

20 Funding

21 This research did not receive any specific grant from funding agencies in the public,

22 commercial, or not-for-profit sectors.

\section{Role of funding source}

25 None 
Addis, D.R., McAndrews, M.P., 2006. Prefrontal and hippocampal contributions to the generation and binding of semantic associations during successful encoding. Neuroimage 33, 1194-1206. https://doi.org/10.1016/j.neuroimage.2006.07.039

Adnan, A., Barnett, A., Moayedi, M., McCormick, C., Cohn, M., McAndrews, M.P., 2016. Distinct hippocampal functional networks revealed by tractography-based parcellation. Brain Struct. Funct. 221, 2999-3012. https://doi.org/10.1007/s00429015-1084-x

Aron, A.R., Robbins, T.W., Poldrack, R.A., 2014. Inhibition and the right inferior frontal cortex: One decade on. Trends Cogn. Sci. 18, 177-185.

https://doi.org/10.1016/j.tics.2013.12.003

Banks, S.J., Eddy, K.T., Angstadt, M., Nathan, P.J., Luan Phan, K., 2007. Amygdalafrontal connectivity during emotion regulation. Soc. Cogn. Affect. Neurosci. 2, 303312. https://doi.org/10.1093/scan/nsm029

Bargh, J.A., Williams, L.E., 2007. The case for nonconscious emotion regulation., in: Gross, J.J. (Ed.), Handbook of Emotion Regulation. Guilford Press, New York, NY, pp. 429-445.

Berking, M., Wirtz, C.M., Svaldi, J., Hofmann, S.G., 2014. Emotion regulation predicts symptoms of depression over five years. Behav. Res. Ther. 57, 13-20. https://doi.org/10.1016/j.brat.2014.03.003

Botvinick, M.M., Cohen, J.D., Carter, C.S., 2004. Conflict monitoring and anterior cingulate cortex: an update. Trends Cogn. Sci. 8, 539-546.

Brown, J.M., 2013. A sex difference in location-based inhibition-of-return. Pers. Individ. Dif. 54, 721-725. https://doi.org/10.1016/j.paid.2012.11.029

Corbetta, M., Shulman, G.L., 2002. Control of goal-directed and stimulus-driven attention in the brain. Nat. Rev. Neurosci. 3, 201-215. https://doi.org/10.1038/nrn755

Dalili, M.N., Penton-Voak, I.S., Harmer, C.J., Munafò, M.R., 2015. Meta-analysis of emotion recognition deficits in major depressive disorder. Psychol. Med. 45, 11351144. https://doi.org/10.1017/S0033291714002591

Dan, R., Canetti, L., Keadan, T., Segman, R., Weinstock, M., Bonne, O., Reuveni, I., Goelman, G., 2019. Sex differences during emotion processing are dependent on the menstrual cycle phase. Psychoneuroendocrinology 100, 85-95. https://doi.org/10.1016/j.psyneuen.2018.09.032

Depue, B.E., Orr, J.M., Smolker, H.R., Naaz, F., Banich, M.T., 2016. The Organization of Right Prefrontal Networks Reveals Common Mechanisms of Inhibitory Regulation Across Cognitive, Emotional, and Motor Processes. Cereb. Cortex 26, 1634-1646. https://doi.org/10.1093/cercor/bhu324

Domes, G., Schulze, L., Böttger, M., Grossmann, A., Hauenstein, K., Wirtz, P.H., Heinrichs, M., Herpertz, S.C., 2010. The neural correlates of sex differences in emotional reactivity and emotion regulation. Hum. Brain Mapp. 31, 758-769. https://doi.org/10.1002/hbm.20903

Etkin, A., Egner, T., Peraza, D.M., Kandel, E.R., Hirsch, J., 2006. Resolving Emotional Conflict: A Role for the Rostral Anterior Cingulate Cortex in Modulating Activity in the Amygdala. Neuron 51, 871-882. https://doi.org/10.1016/j.neuron.2006.07.029 
Fabes, R.A., Martin, C.L., 1991. Gender and Age Stereotypes of Emotionality. Personal. Soc. Psychol. Bull. 17, 532-540. https://doi.org/10.1177/0146167291175008

Fan, J., Flombaum, J.I., McCandliss, B.D., Thomas, K.M., Posner, M.I., 2003. Cognitive and brain consequences of conflict. Neuroimage 18, 42-57.

Faul, F., Erdfelder, E., Lang, A.G., Buchner, A., 2007. G*Power 3: A flexible statistical power analysis program for the social, behavioral, and biomedical sciences. Behav. Res. Methods 39, 175-191. https://doi.org/10.3758/BF03193146

Fischer, A.H., 1993. Sex Differences in Emotionality: Fact or Stereotype? Fem. Psychol. 3, 303-318. https://doi.org/10.1177/0959353593033002

Frank, D.W., Dewitt, M., Hudgens-Haney, M., Schaeffer, D.J., Ball, B.H., Schwarz, N.F., Hussein, A.A., Smart, L.M., Sabatinelli, D., 2014. Emotion regulation: Quantitative meta-analysis of functional activation and deactivation. Neurosci. Biobehav. Rev. 45, 202-211. https://doi.org/10.1016/j.neubiorev.2014.06.010

Gard, M.G., Kring, A.M., 2007. Sex differences in the time course of emotion. Emotion. https://doi.org/10.1037/1528-3542.7.2.429

Garnefski, N., Teerds, J., Kraaij, V., Legerstee, J., van den Kommer, T., 2004. Cognitive emotion regulation strategies and depressive symptoms: Differences between males and females. Pers. Individ. Dif. 36, 267-276. https://doi.org/10.1016/S01918869(03)00083-7

Giombini, L., 2015. Handbook of emotion regulation, Advances in Eating Disorders. https://doi.org/10.1080/21662630.2014.953321

Green, M.J., Cahill, C.M., Malhi, G.S., 2007. The cognitive and neurophysiological basis of emotion dysregulation in bipolar disorder. J. Affect. Disord. 103, 29-42. https://doi.org/10.1016/j.jad.2007.01.024

Greenberg, D.L., Rice, H.J., Cooper, J.J., Cabeza, R., Rubin, D.C., LaBar, K.S., 2005. Co-activation of the amygdala, hippocampus and inferior frontal gyrus during autobiographical memory retrieval. Neuropsychologia 43, 659-674. https://doi.org/10.1016/j.neuropsychologia.2004.09.002

Gross, J.J., 2015. Emotion Regulation: Current Status and Future Prospects. Psychol. Inq. 26, 1-26. https://doi.org/10.1080/1047840X.2014.940781

Grossman, M., Wood, W., 1993. Sex Differences in Intensity of Emotional Experience: A Social Role Interpretation. J. Pers. Soc. Psychol. 65, 1010-1022. https://doi.org/10.1037/0022-3514.65.5.1010

Gur, R.C., 2002. Sex Differences in Temporo-limbic and Frontal Brain Volumes of Healthy Adults. Cereb. Cortex 12, 998-1003. https://doi.org/10.1093/cercor/12.9.998

Hampson, E., van Anders, S.M., Mullin, L.I., 2006. A female advantage in the recognition of emotional facial expressions: test of an evolutionary hypothesis. Evol. Hum. Behav. 27, 401-416. https://doi.org/10.1016/j.evolhumbehav.2006.05.002

Hess, U., Senecal, S., Kirouac, G., Herrera, P., Philippot, P., Kleck, R.E., 2000. Emotional expressivity in men and women: Stereotypes and self-perceptions. Cogn. Emot. 14, 609-642. https://doi.org/10.1080/02699930050117648

Howard, L.R., Kumaran, D., Ólafsdóttir, H.F., Spiers, H.J., 2013. Dissociation between Dorsal and Ventral Posterior Parietal Cortical Responses to Incidental Changes in Natural Scenes. PLoS One 8, e67988-e67988. 
https://doi.org/10.1371/journal.pone.0067988

Joormann, J., Stanton, C.H., 2016. Examining emotion regulation in depression: A review and future directions. Behav. Res. Ther. https://doi.org/10.1016/j.brat.2016.07.007

Kessler, R.C., McGonagle, K.A., Swartz, M., Blazer, D.G., Nelson, C.B., 1993. Sex and depression in the National Comorbidity Survey I: Lifetime prevalence, chronicity and recurrence. J. Affect. Disord. 29, 85-96. https://doi.org/10.1016/01650327(93)90026-G

Kohn, N., Eickhoff, S.B., Scheller, M., Laird, A.R., Fox, P.T., Habel, U., 2014. Neural network of cognitive emotion regulation - An ALE meta-analysis and MACM analysis. Neuroimage 87, 345-355. https://doi.org/10.1016/j.neuroimage.2013.11.001

Kong, F., Zhen, Z., Li, J., Huang, L., Wang, X., Song, Y., Liu, J., 2014. Sex-related neuroanatomical basis of emotion regulation ability. PLoS One 9, e97071-e97071. https://doi.org/10.1371/journal.pone.0097071

Konishi, S., Nakajima, K., Uchida, I., Kikyo, H., Kameyama, M., Miyashita, Y., 1999. Common inhibitory mechanism in human inferior prefrontal cortex revealed by event-related functional MRI. Brain 122, 981-991. https://doi.org/10.1093/brain/122.5.981

Kring, A.M., Gordon, A.H., 1998. Sex Differences in Emotion: Expression, Experience, and Physiology. J. Pers. Soc. Psychol. https://doi.org/10.1037/0022-3514.74.3.686

Lang, P., Bradley, M., Cuthbert, B., 2005. International Affective Picture System (IAPS): Digitized Photographs, Instruction Manual and Affective Ratings. Technical Report A-6., 2005.

Lau, H., Rogers, R.D., Passingham, R.E., 2006. Dissociating response selection and conflict in the medial frontal surface. Neuroimage 29, 446-451. https://doi.org/10.1016/j.neuroimage.2005.07.050

Leach, L.S., Christensen, H., Mackinnon, A.J., Windsor, T.D., Butterworth, P., 2008. Gender differences in depression and anxiety across the adult lifespan: The role of psychosocial mediators. Soc. Psychiatry Psychiatr. Epidemiol. 43, 983-998. https://doi.org/10.1007/s00127-008-0388-z

$\mathrm{Li}, \mathrm{H}$. , Yuan, J., Lin, C., 2008. The neural mechanism underlying the female advantage in identifying negative emotions: An event-related potential study. Neuroimage 40, 1921-1929. https://doi.org/10.1016/j.neuroimage.2008.01.033

Linhartová, P., Látalová, A., Kóša, B., Kašpárek, T., Schmahl, C., Paret, C., 2019. fMRI neurofeedback in emotion regulation: A literature review. Neuroimage 193, 75-92. https://doi.org/10.1016/j.neuroimage.2019.03.011

Lungu, O., Potvin, S., Tikàsz, A., Mendrek, A., 2015. Sex differences in effective frontolimbic connectivity during negative emotion processing. Psychoneuroendocrinology 62, 180-188. https://doi.org/10.1016/j.psyneuen.2015.08.012

Mak, A.K.Y., Hu, Z. guo, Zhang, J.X.X., Xiao, Z., Lee, T.M.C., 2009. Sex-related differences in neural activity during emotion regulation. Neuropsychologia 47 , 2900-2908. https://doi.org/10.1016/j.neuropsychologia.2009.06.017

Mauss, I.B., Bunge, S.A., Gross, J.J., 2007. Automatic Emotion Regulation. Soc. Personal. Psychol. Compass 1, 146-167. https://doi.org/10.1111/j.17519004.2007.00005.x 
McRae, K., Ochsner, K.N., Mauss, I.B., Gabrieli, J.J.D., Gross, J.J., 2008. Gender differences in emotion regulation: An fMRI study of cognitive reappraisal. Gr. Process. Intergr. Relations 11, 143-162. https://doi.org/10.1177/1368430207088035

Nieto-Castanon, A., 2015a. NITRC: CONN : functional connectivity toolbox: RE: Event Related Analyses with CONN [WWW Document]. URL https://www.nitrc.org/forum/message.php?msg_id=12696 (accessed 2.26.20).

Nieto-Castanon, A., 2015b. NITRC: CONN : functional connectivity toolbox: RE: ROI-toROI F tests. [WWW Document]. URL https://www.nitrc.org/forum/message.php?msg_id=13535 (accessed 2.21.20).

Nolen-Hoeksema, S., 2012. Emotion Regulation and Psychopathology: The Role of Gender. Annu. Rev. Clin. Psychol. 8, 161-187. https://doi.org/10.1146/annurevclinpsy-032511-143109

Nolen-Hoeksema, S., 2001. Gender differences in depression. Curr. Dir. Psychol. Sci. https://doi.org/10.1111/1467-8721.00142

O’Driscoll, C., Laing, J., Mason, O., 2014. Cognitive emotion regulation strategies, alexithymia and dissociation in schizophrenia, a review and meta-analysis. Clin. Psychol. Rev. 34, 482-495. https://doi.org/10.1016/j.cpr.2014.07.002

Ochsner, K.N., Gross, J.J., 2005. The cognitive control of emotion. Trends Cogn. Sci. 9, 242-249. https://doi.org/10.1016/j.tics.2005.03.010

Ochsner, K.N., Silvers, J.A., Buhle, J.T., 2012. Functional imaging studies of emotion regulation: a synthetic review and evolving model of the cognitive control of emotion. Ann. N. Y. Acad. Sci. 1251, E1-E24. https://doi.org/10.1111/j.17496632.2012.06751.x

Ohira, H., Nomura, M., Ichikawa, N., Isowa, T., lidaka, T., Sato, A., Fukuyama, S., Nakajima, T., Yamada, J., 2006. Association of neural and physiological responses during voluntary emotion suppression. Neuroimage 29, 721-733. https://doi.org/10.1016/j.neuroimage.2005.08.047

Penny, W., Friston, K., Ashburner, J., Kiebel, S., Nichols, T., 2007. Statistical Parametric Mapping: The Analysis of Functional Brain Images, Statistical Parametric Mapping: The Analysis of Functional Brain Images. https://doi.org/10.1016/B978-0-12-372560-8.X5000-1

Phillips, M.L., Drevets, W.C., Rauch, S.L., Lane, R., 2003. Neurobiology of emotion perception II: Implications for major psychiatric disorders. Biol. Psychiatry 54, 515528. https://doi.org/10.1016/S0006-3223(03)00171-9

Phillips, M.L., Ladouceur, C.D., Drevets, W.C., 2008. A neural model of voluntary and automatic emotion regulation: Implications for understanding the pathophysiology and neurodevelopment of bipolar disorder. Mol. Psychiatry. https://doi.org/10.1038/mp.2008.65

Picó-Pérez, M., Radua, J., Steward, T., Menchón, J.M., Soriano-Mas, C., 2017. Emotion regulation in mood and anxiety disorders: A meta-analysis of $\mathrm{fMRI}$ cognitive reappraisal studies. Prog. Neuro-Psychopharmacology Biol. Psychiatry. https://doi.org/10.1016/j.pnpbp.2017.06.001

Pizzagalli, D.A., 2011. Frontocingulate dysfunction in depression: toward biomarkers of treatment response. Neuropsychopharmacology 36, 183-206. https://doi.org/10.1038/npp.2010.166 
Plant, E.A., Hyde, J.S., Keltner, D., Devine, P.G., 2000. The gender stereotyping of emotions. Psychol. Women Q. 24, 81-92. https://doi.org/10.1111/j.14716402.2000.tb01024.x

Ritchie, S.J., Cox, S.R., Shen, X., Lombardo, M. V., Reus, L.M., Alloza, C., Harris, M.A., Alderson, H.L., Hunter, S., Neilson, E., Liewald, D.C.M., Auyeung, B., Whalley, H.C., Lawrie, S.M., Gale, C.R., Bastin, M.E., McIntosh, A.M., Deary, I.J., 2018. Sex differences in the adult human brain: Evidence from 5216 UK biobank participants. Cereb. Cortex 28, 2959-2975. https://doi.org/10.1093/cercor/bhy109

Stevens, F.L., Hurley, R.A., Taber, K.H., 2011. Anterior cingulate cortex: Unique role in cognition and emotion. J. Neuropsychiatry Clin. Neurosci. 23, 121-125. https://doi.org/10.1176/jnp.23.2.jnp121

Stoet, G., 2010. Sex differences in the processing of flankers. Q. J. Exp. Psychol. 63, 633-638. https://doi.org/10.1080/17470210903464253

Stuss, D.T., Levine, B., 2002. Adult Clinical Neuropsychology: Lessons from Studies of the Frontal Lobes. Annu. Rev. Psychol. 53, 401-433. https://doi.org/10.1146/annurev.psych.53.100901.135220

Thayer, R.E., Newman, J.R., McClain, T.M., 1994. Self-Regulation of Mood: Strategies for Changing a Bad Mood, Raising Energy, and Reducing Tension, Journal of Personality and Social Psychology. https://doi.org/10.1037/0022-3514.67.5.910

Thomsen, D.K., Mehlsen, M.Y., Viidik, A., Sommerlund, B., Zachariae, R., 2005. Age and gender differences in negative affect - Is there a role for emotion regulation? Pers. Individ. Dif. 38, 1935-1946. https://doi.org/10.1016/j.paid.2004.12.001

Timmers, M., Fischer, A.H., Manstead, A.S.R., 2003. Ability versus vulnerability: Beliefs about men's and women's emotional behaviour. Cogn. Emot. https://doi.org/10.1080/02699930302277

Viviani, R., 2013. Emotion regulation, attention to emotion, and the ventral attentional network. Front. Hum. Neurosci. https://doi.org/10.3389/fnhum.2013.00746

Weilbächer, R.A., Gluth, S., 2017. The interplay of hippocampus and ventromedial prefrontal cortex in memory-based decision making. Brain Sci. https://doi.org/10.3390/brainsci7010004

Weissman, D.H., Gopalakrishnan, A., Hazlett, C.J., Woldorff, M.G., 2005. Dorsal anterior cingulate cortex resolves conflict from distracting stimuli by boosting attention toward relevant events. Cereb. cortex 15, 229-237.

Whitfield-Gabrieli, S., Nieto-Castanon, A., 2012. Conn: A Functional Connectivity Toolbox for Correlated and Anticorrelated Brain Networks. Brain Connect. 2, 125141. https://doi.org/10.1089/brain.2012.0073

Whittle, S., Yücel, M., Yap, M.B.H., Allen, N.B., 2011. Sex differences in the neural correlates of emotion: Evidence from neuroimaging. Biol. Psychol. https://doi.org/10.1016/j.biopsycho.2011.05.003

Wu, Y., Li, H., Zhou, Y., Yu, J., Zhang, Y., Song, M., Qin, W., Yu, C., Jiang, T., 2016. Sex-specific neural circuits of emotion regulation in the centromedial amygdala. Sci. Rep. 6. https://doi.org/10.1038/srep23112

Yamasaki, H., LaBar, K.S., McCarthy, G., 2002. Dissociable prefrontal brain systems for attention and emotion. Proc. Natl. Acad. Sci. U. S. A. 99, 11447-11451. https://doi.org/10.1073/pnas.182176499 